without this and cultivation on nutrient media, and the reproduction of the disease from culture, it would be unwise to pronounce as to their nature.

The larger spherical bodies, described by MI. Laveran as slightly larger than red blood cells, I have not hitherto seen; but $1 \mathrm{am}$ inclined to believe that, what are described by him as the larger spheroids are red blood cells invaded by the parasitical elements I have observed. The white blood-corpuscles in malaria are not increased; they are apparently little affected by the disease, being invaded occasionally by the nucleated spheroids; and, when studded with pigment granules, as sometimes occurs, they present the appearance indicated by $\mathbf{M}$. Laveran as the "leucocytes mélaniferes."

\section{THE CIIEMICAL INCOMPATIBILITY OF ANTISEPTIC AGENTS.}

BY ROBERT BOXALL, M.D., M.R.C.l'.,

Physician to the General Lying-in and to the Samaritan Free Hospitals.

THE necessity of employing antiseptic agents in solution of definite strength will be, $I$ presume, on all hands conceded; for, if the solution be too attenuated, the object in view will fail in its accomplishment, and, if too concentrated, considerable damage will in many cases be wrought, not only locally on the tissues to which the application is made, but also on the body generally as the result of absorption. The borderland between safety and success is, in many instances, a very narrow one. The possibility of reducing the strength of the solution, or of altering its nature through the chemical incompatibility of the materials employed, has hitherto received but little attention. The important practical bearing which this may exert on their efficiency as antiseptics must prove my apology for drawing attention to the matter.

By way of example, I have selected five of the more important antiseptic agents in general use, and, for ready reference as to the incompatibilities of each, the results of the experiments are presented in a tabular form, showing the action not only of these agents on one another, but also of certain lubricants with which they are frequently combined and brought into contact, and of soap with which they are apt to be contaminated in the process of washing and disinfecting the hands and instruments.

In view of the practical utility of these observations, the experiments were made, not with concentrated materials, but with solutions of the strengths usually employed in practice, and were carried out at temperatures not exceeding that of the body.
1. Corrosive Sublimate Solution (Perchloride of Mercury)

. Carbolic Solution (Phenol) $\ldots \ldots$ of Potassium)

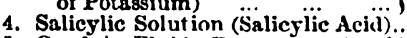

5. Condy's Fluid (Permanganate of $j$ Potassium

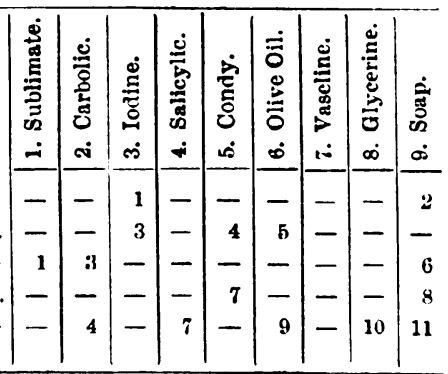

The following incompatibilities were observed :

1. Corrosive Sublimate and Iodine.-No precipitate of mercuric iodide is at any stage of the admixture formed. A small addition of sublimate solution fixes the free iodine. as may be seen by the immediate bleaching of the iodine solution, and confirmed by the subsequent addition of starch paste, which produces no blue coloration. One part by volume of sublimate solution $(1$ in 1,000$)$ is just sufficient to fix the whole of the free iodine in 4 parts by volume of iodine solution (tr. iod. B.P. $3 \mathrm{j}$ in $\mathrm{Oj}$ ). N.B.-This forms a rough and ready test for the strength of sublimute solutions.

2. Corrosive Sublimate and Suap.-An insoluble soap is produced even when a neutral soap solution is used. This is of special importance in consideration of the small admixture with soap which is required to throw down the whole of the mercury from solutions of the strength usually employed.

3. Carbolic and Iodine.-An exceedingly small admixture with phenol is sufficient to fix the whole of the free iodine as in (1). One part by volume of carbolic solution (1 in 20) removes the whole of the free iodine from 2,000 parts by volume of iodine solution of the strength indicated above.

4. Carbolic and Condy.-This is perhaps the most generally recognised of these incompatibilities. Admixture with phenol immediately turns permanganate brown.

5. Carbolic and Olive Oil.-This is of importance and of special interest when taken in conjunction with the researches of Koch, of Berlin, who has shown that bacillus spores are capable of living and developing after having been immersed in carbolised oil (1 in 20) for 4 months. The oil appears to enter into some combination with and to fix the phenol. If a drop of tr. ferri perchlor. B.P. be shaken up in a test tube with carbolised oil ( 1 in 20$)$ no change is found to have been produced in the iron as it gravitates to the bottom. Moreover, if carbolised oil be shaken up with a few drops of water, the water allowed to separate out at the bottom of the tube and a drop of iron solution conveyed into it, the characteristic purple coloration with phenol is not produced unless the shaking has been very prolonged and energetic, and then only to a slighit degree. By strongly heating the carbolised oil phenol is again set free, and the above reaction can then be obtained.

6. Iodine and Soap.-No action is produced by a neutral soap solution, but ordinary soap, which contains an excess of alkali, at once removes the free iodine.

7. Salicylic Acid and Condy.-A very dilute salicylic acid solution ( 1 in 800) slowly removes the colour from permanganate.

8. Salicylic Acid and Soap.-A drop of dilute salicylic acid solution gives a white precipitate even when a neutral soap solution is emplojed.

9. Condy and Olive Oil.- When permanganate solution is shaken up with olive oil its violet colour is changed to brown.

10. Condy and Glycerine.- When permanganate solution is added to glycerine its colour slowly changes.

11. Condy and Soap.-This incompatibility is also generally recognised. Soap, even when a neutral solution is employed, readily turns permanganate brown.

I do not pretend to any precise knowledge of the bodies produced, some of which may, for all I know, possess powerful antiseptic properties. But until this point is settled by direct observation, when chemical incompatibility exists, the antiseptic properties of the original solution must be regarded as weakened, if not wholly destroyed.

The moral conreyed by the above experiments is obvious : avoid as far as possible the admixture of antiseptic agents and their contamination with lubricants and with soap when incompatibility exists. For instance, in employing corrosive sublimate it is advisable to use the same solution for disinfecting the hands (carefully aroiding contamination with soap) and for cleansing instruments ${ }^{1}$ as for irrigating the parts, to employ a mercurialised lubricant and to use alembroth dressings. If for any reason it becomes requisite to substitute one antiseptic agent for another, a second should be chosen which is not incompatible with the first, and the same precautions should be observed throughout the series.

The above observations deal with one phase only of the subject. I am content to leave to more able hands the elaboration of further details. The second question-the chemical nature of the bodies produced -is for the chemist to answer; the third point-the antiseptic value of these bodies-still remains for the germiculturist to determine. When these points have been settled and acted upon, less scepticism as to the value of antiseptic agents mny be looked for. The fuult lies not so much with the antiseptics themselves as with the unscientific method in which they are often employed.

1 Here, again, a caution is requisite, for copper and steel, unless nickel plated. are apt to decompose the solution and to cause precipitation of the mercury in a free state.

TestruoniaL.-Dr. Williams, who was recently compelled to resign his appointment of medical superintendent of the Sussex Lunatic Asylum at Hayward's Heath in consequence of ill-health, has been presented by upwards of one hundred members of the staff of that institution with an illuminated address in the form of an album as an expression of their apprecintion and regard.

At a meeting of the Committee of the Home and Infirmary for Sick Children, Lower Sydenham, S.E., on April 2nd, a unanimous vote of thanks was passed to Dr. Spencer Smyth, F.R.C.S. for valuable services rendered to the institution for so long a time as Honorary Surgeon and Consulting Surgeon. Dr. Smyth has gono to reside at Bournemouth. 